\title{
Análise de fatores de risco envolvidos na falta de conhecimento materno sobre o teste de triagem neonatal
}

\author{
Analysis of risk factors involved in lack \\ of maternal knowledge about neonatal \\ screening
}

\section{RESUMO}

Introdução: a triagem neonatal, conhecida popularmente como 'teste do pezinho', tem a finalidade de detectar diversas patologias no recém-nascido antes do aparecimento de sintomas. Objetivo: analisar o conhecimento materno acerca da triagem neonatal e os fatores de risco relacionados à falta de informação sobre o tema. Métodos: estudo prospectivo, transversal, por meio da aplicação de um questionário em puérperas situadas em maternidades públicas entre 2013 a 2015. Testes de Mann-Whitney e qui-quadrado foram utilizados para verificar associações entre as variáveis categóricas e contínuas. Resultados: foram validados 227 questionários. A maioria das puérperas teve seis ou mais consultas de pré-natal, sendo que 3,5\% não tiveram nenhuma assistência pré-natal. Quando questionadas sobre o 'teste do pezinho', 90,7\% afirmaram não ter recebido nenhuma informação durante o pré-natal e 17,2\% das participantes não tinham conhecimento sobre o tema. Falta de conhecimento sobre a triagem neonatal foi associada com idade inferior a 18 anos, baixo nível de escolaridade, primigestas e mães em maternidades de alta complexidade. Conclusão: ficou patente a falha do pré-natal na orientação sobre essa intervenção. Considerando a importância da triagem neonatal, confirma-se a necessidade de aumentar a passagem de informação para as gestantes, especialmente aquelas em grupos de risco.

Palavras-chave: Triagem neonatal; Conhecimento; Educação; Período pós-parto.
Mariana Fernandes Guimarães

Graduanda na Faculdade de Medicina da Universidade Federal Fluminense, Departamento Materno-Infantil.

\section{Fernanda Aniceto Pereira e Rabelo}

Graduanda na Faculdade de Medicina da Universidade Federal Fluminense, Departamento Materno-Infantil.

\section{Israel Figueiredo Junior}

Professor-Associado do Departamento Materno-Infantil da Faculdade de Medicina da Universidade Federal Fluminense.

\section{Instituição:}

Universidade Federal Fluminense

\section{Correspondência:}

mari.fguimaraes@gmail.com

Recebido em: 13/12/2017

Aprovado em: 4/1/2018 


\section{ABSTRACT}

Introduction: neonatal screening, popularly known as 'heel-stick test', is designed to detect various pathologies in newborns before the onset of symptoms. Objective: to assess maternal knowledge of neonatal screening and risk factors associated with lack of information. Methods: a cross-sectional prospective study developed through the application of a questionnaire with breastfeeding women recruited from public maternity units between 2013 and 2015. Mann-Whitney and chi-square tests were used to verify associations between categorical and continuous variables. Results: 227 questionnaires were validated. Most participants had six or more prenatal appointments, and 3.5\% had no prenatal care. When questioned about the 'heel-stick test', $90.7 \%$ claimed receiving no information about it during prenatal visits and $17.2 \%$ had no knowledge about the topic. Lack of knowledge about neonatal screening was associated with women aged less than 18 years, low education level, primiparity and delivery at high-complexity facilities. Conclusion: prenatal care failed to provide orientation about this intervention. The importance of neonatal screening attests the need to improve guidance to pregnant women, especially those in high-risk groups.

Keywords: Neonatal screening; Knowledge; Education; Postpartum period.

\section{INTRODUÇÃO}

A triagem neonatal (TN), conhecida popularmente como 'teste do pezinho', é um conjunto de exames preventivos laboratoriais realizados em amostras de sangue coletadas por meio de uma leve punção do calcanhar do recém-nascido. Tem como finalidade detectar erros inatos do metabolismo antes mesmo do aparecimento de sintomas. ${ }^{1,2}$ Caso essas doenças não sejam diagnosticadas e tratadas precocemente, podem desencadear sérias consequências com repercussões na vida adulta, incluindo sequelas neurológicas e outras complicações. ${ }^{3-5}$ A coleta deve ser feita preferencialmente entre o terceiro e sétimo dia de vida. ${ }^{2}$ A realização do teste antes de 48 horas deve ser evitada, devido ao risco de resultados falso-negativos. ${ }^{1}$

A TN foi proposta nos Estados Unidos pelo Dr. Robert Guthrie em 1963. ${ }^{6}$ Inicialmente, o teste detectava concentrações séricas do aminoácido fenilalanina, que encontram-se elevadas na fenilcetonúria. Nos anos seguintes, programas de TN expandiram para diversos países e novas patologias foram incluídas.

No Brasil, a TN para fenilcetonúria começou em 1976, com o pediatra Dr. Benjamin José Schmidt ${ }^{7}$ e em 1980 foi introduzida a triagem para o hipotireoidismo congênito. ${ }^{8}$ Em 2001, foi criado o Programa Nacional de Triagem Neonatal (PNTN), um programa de saúde pública que possuía como principais objetivos ampliar o número de doenças detectadas pelo exame e expandir a cobertura populacional, estabelecendo como meta alcançar 100\% dos nascidos vivos. ${ }^{1}$ Hoje, oficialmente, o Sistema Único de Saúde do Brasil proporciona a cobertura obrigatória da TN para fenilcetonúria, hipotireoidismo congênito, doença falciforme e outras hemoglobinopatias, fibrose cística, hiperplasia adrenal congênita e deficiência de biotinidase no escopo do programa. ${ }^{1}$ Entretanto, no setor privado, inúmeras patologias (entre 40-50) podem ser rastreadas no período neonatal com sangue extraído do cordão umbilical.

De acordo com dados do Ministério da Saúde, no ano anterior ao lançamento do PNTN, a cobertura da TN no Brasil era em torno de 55\%; em 2005, essa taxa foi estimada para aproximadamente $80 \% .^{9,10}$ Dados mais recentes mostram que os testes gratuitos cobrem $76,91 \%$ dos nascidos vivos no Brasil. Dos quase 3 milhões de nascimentos por ano, ${ }^{1}$ em 2016, mais de 2,3 milhões de recém-nascidos fizeram o teste do pezinho em todo o país. ${ }^{11}$ Apesar desse aumento significativo após a criação do PNTN, observa-se grande heterogeneidade entre as diversas regiões do país e as taxas de cobertura ainda encontram-se aquém do esperado em algumas localidades. Além disso, alguns estudos 
demonstraram que o teste vem sendo realizado tardiamente, o que pode atrasar o início do tratamento e, consequentemente, dificultar a prevenção de sequelas. ${ }^{10}$

Como muitos berçários e maternidades não apresentam a infraestrutura e os materiais necessários para a coleta do exame, a responsabilidade de levar seus filhos ao posto de saúde para realizar a TN, após receber alta da maternidade, é repassada aos pais. Dessa maneira, para que esta seja realizada corretamente e as metas de cobertura populacional sejam atingidas, é fundamental a ampla implementação e informação do tema durante o pré-natal, ${ }^{12}$ estabelecendo contatos suficientes com as mães para introduzir o conceito do sistema de triagem e como este funciona.

Essa pesquisa levantou a hipótese de desconhecimento e/ou desinformação dos pais em relação à triagem neonatal. O estudo teve como objetivos pesquisar o entendimento das mulheres no período pós-parto sobre a TN e identificar fatores de risco maternos e gestacionais associados à falta de conhecimento sobre essa intervenção, além de avaliar a oferta de informações sobre o tema durante as consultas do pré-natal.

\section{MÉTODO}

Este módulo descreve de forma breve todos os passos percorridos, desde a coleta de dados até o tratamento estatístico, para a efetivação da pesquisa.

\section{Desenho do estudo}

Trata-se de uma pesquisa prospectiva, transversal e com aplicação de questionário. A amostra foi constituída por puérperas voluntárias, recrutadas de 2013 a 2015. Todas forneceram consentimento informado por escrito para a participação. O protocolo do estudo foi aprovado pelo Comitê de Ética em Pesquisa da Universidade Federal Fluminense (CAAE: 23136714.7.0000.5243).

\section{LoCAIS DE REALIZAÇÃo}

Este estudo foi realizado em três maternidades públicas do Município de Niterói, Estado do Rio de Janeiro, Brasil. Duas eram instalações de alta complexidade $(3 \mathrm{~A} ; 3 \mathrm{~B})$, enquanto a terceira era uma unidade de baixa complexidade (2A).

\section{Participantes}

Puérperas que estavam interessadas no tema foram incluídas no estudo, independentemente de ter recebido atenção pré-natal adequada ou informações específicas sobre a TN. Os locais e datas de coleta de dados foram escalonados para maximizar a cobertura. As mulheres foram selecionadas aleatoriamente usando uma lista de números gerada por um software estatístico. Ao final do questionário, cada participante recebeu uma brochura informativa sobre o tema. Três mulheres que se recusaram a preencher o questionário foram excluídas da análise.

\section{TAMANHO do ESTUdo, FONTE DE DADOS, MENSURAÇÕES}

Para avaliar o questionário do estudo, foi realizado um teste-piloto em uma amostra de 25 puérperas. Esta fase durou seis meses e os dados das participantes foram excluídos devido a alterações no conteúdo do instrumento.

A versão definitiva foi administrada durante um período de aproximadamente um ano e meio. O banco de dados final compreendeu 227 entrevistadas. O questionário foi administrado por dois dos autores previamente treinados e sempre trabalhados em par para minimizar o viés intra e interobservador.

\section{VARIÁVEIS DE INTERESSE}

foram analisadas variáveis maternas, gestacionais, periparto e neonatais. As mulheres foram entrevistadas quanto ao município de residência, idade, nível de escolaridade, número de gestações, número de consultas pré-natais, conhecimento sobre a TN e se foram fornecidas informações sobre o tema durante as consultas pré-natais. Nenhuma informação sobre raça ou cor da pele foi coletada. Devido à ampla diversidade genômica da população brasileira, a definição de ascendência com base na cor da pele é conhecida como inexata. $^{13}$

De acordo com o número de consultas pré-natais realizadas, as participantes foram estratificadas em três grupos: nenhuma visita; até seis visitas; ou mais de seis visitas. As participantes também foram classificadas, de acordo com o número de gestações, como primigestas (mulheres que estavam grávidas pela primeira vez) ou multigestas (mulheres que já estiveram grávidas antes, independentemente de o feto ter sobrevivido). A escolaridade foi classificada em quatro categorias: Ensino Fundamental incompleto (menos de oito anos de educação formal, incluindo analfabetas); Ensino Fundamental completo (oito anos); Ensino Médio (9 a 11 anos) e Ensino Superior (mais de 11 anos). ${ }^{14}$

Para os propósitos deste estudo, de acordo com suas respostas ao questionário, as participantes foram divididas em dois grupos: com conhecimento (CC) e sem conhecimento (SC). O grupo SC compreendia aquelas participantes que alegaram nunca ter ouvido falar sobre a TN, ou que tinham ouvido falar, mas não tinham conhecimento sobre os seus objetivos, o prazo ideal para a sua realização e a gravidade das doenças envolvidas. Ao longo do questionário, o termo 'teste do pezinho' foi 
usado para se referir à triagem neonatal, para maior facilidade de compreensão pelas participantes.

Os seguintes dados foram recuperados a partir de prontuários: idade gestacional; Apgar de 5 minutos; via de parto; sexo do recém-nascido; e peso ao nascer. Além disso, os documentos maternos e neonatais foram utilizados para verificar os dados coletados diretamente durante as entrevistas com as participantes.

\section{MÉtodo estatístico}

Foi realizada uma análise descritiva dos dados coletados. As variáveis categóricas foram expressas como frequências absolutas e relativas. Para as variáveis quantitativas (idade materna, número de gestações, número de consultas pré-natais, peso, idade gestacional e escore de Apgar) foram calculadas a média, a amplitude, o desvio-padrão (DP) e o intervalo de confiança (IC). Após a verificação de distribuição normal da amostra, foram utilizados métodos não paramétricos para testar as hipóteses propostas. O Teste de Mann-Whitney foi usado para comparar médias ranqueadas entre os dois grupos (CC e SC).

Utilizou-se o teste qui-quadrado com o teste exato de Fisher para avaliar as relações entre cada grupo e as seguintes variáveis categóricas: parto em unidade de maternidade de baixa complexidade ou alta complexidade $(0 / 1)$; município de residência Niterói ou outro $(0 / 1)$; idade - menor de 18 ou maior de 18 anos $(0 / 1)$; primigesta ou não primigesta $(0 / 1)$; número de consultas pré-natais - menor que 6 ou maior que $6(0 / 1)$; escolaridade menor ou igual a 8 anos (analfabetas, Ensino Fundamental completo ou incompleto) ou acima de 8 anos (Ensino Médio incompleto, completo ou Ensino Superior); e via de parto - vaginal ou cesariana $(0 / 1)$. As razões de chance (odds ratios - OR) também foram calculadas para os testes de associação. A significância estatística foi aceita em p-valor: menor que 0,05 para todos os testes.

\section{RESULTADOS}

Os 227 questionários validados foram coletados na maternidade $2 \mathrm{~A}(34,8 \%$, $79 / 227)$, hospital 3A $(23,8 \%, 54 / 227)$ e hospital 3B (41,4\%, 94/227). A maioria das participantes era dos municípios de Niterói $(65,2 \%, 148 / 227)$ e São Gonçalo $(25,6 \%$, 58/227). A média de idade materna foi de 24,85 anos (IC95\%: 24,03-25,67, DP: 6,13, amplitude: 14-46). Apenas 20 das 227 entrevistadas $(8,8 \%)$ tinham menos de 18 anos de idade. O nível de escolaridade mais prevalente foi o Ensino Médio (55,5\% , 126/227), seguido pelo Ensino Fundamental incompleto $(32,6 \%$, 74/227), Ensino Fundamental completo $(8,4 \%, 19 / 227)$ e Ensino Superior $(3,5 \%, 8 / 227)$.

A maioria das participantes $(61,2 \%$, $139 / 227$ ) tinha frequentado seis ou mais consultas de cuidados pré-natais. O número médio de consultas foi de 7,26 (IC 95\%: 6,87-7,65 e DP: 2,92). Notavelmente, oito mulheres $(3,5 \%$, 8/227) não tiveram nenhuma assistência pré-natal.

Quanto ao número de gestações, $44,9 \%$ das participantes (102/227) foram primigestas. A cesariana foi a via de parto predominante $(54,2 \%$, $123 / 227)$. O peso médio ao nascer foi de 3.145,71 gramas (IC 95\%: 3.076,613.214,81 e DP: 515,25), e a maioria das crianças era do sexo feminino $(52,4 \%$, 119/227).

Quando questionadas sobre a triagem neonatal, 17,2\% das participantes (39/227) disseram que não tinham conhecimento sobre o teste (SC), e 90,4\% (198/219) das mulheres que receberam assistência pré-natal afirmaram não ter recebido nenhuma informação sobre o tema durante as consultas nessa gestação.

Dentre as participantes que não tiveram assistência pré-natal, somente uma $(12,5 \%, 1 / 8)$ afirmou não ter conhecimento sobre a TN. Neste grupo, das sete restantes, seis mulheres alegaram ter dois ou mais filhos $(85,7 \%$, $6 / 7)$.

Os testes de associações entre as variáveis categóricas e o conhecimento sobre a TN evidenciaram que as mulheres do grupo SC apresentaram maior probabilidade de ter idade menor que 18 anos $(\mathrm{p}<0,01)$, ter baixo nível de escolaridade $(p=0,02)$, ser primigesta $(\mathrm{p}<0,01)$ e estar em uma maternidade de alta complexidade $(\mathrm{p}<0,01)$ (Tabela 1). A análise das variáveis contínuas revelou associações significativas entre idade materna $(\mathrm{p}<0,01)$ e número de gestações $(p<0,01)$ com desconhecimento sobre a TN (Tabela 2 ).

\section{DISCUSSÃo}

Este estudo fornece evidências da contribuição limitada do cuidado pré-natal como um disseminador de informações sobre a TN, visto que a grande maioria das participantes afirmaram ter recebido nenhuma informação durante as consultas naquela gestação. Contudo, conhecimento suficiente foi relatado pela maioria das mulheres na amostra, corroborando estudos anteriores realizados na Arábia Saudita e na Itália. ${ }^{15,16}$ Por outro lado, um estudo americano mostrou que as mulheres e seus familiares gostariam de receber mais informações sobre este tema durante as visitas de cuidados pré-natais. ${ }^{17}$

Um estudo relacionado ao conhecimento materno e às atitudes sobre a TN, conduzido por pesquisadores no Departamento de Pediatria da 
Tabela I - Características das puérperas no estudo de variáveis binárias que influenciam a falta de conhecimento materno sobre a triagem neonatal em Niterói (2013-20I5)

\begin{tabular}{|c|c|c|c|c|c|c|}
\hline VARIÁVEIS BINÁRIAS & Categorias & SC $^{a}$ N. (\%) & $\mathrm{CC}^{\mathrm{b}}$ N. (\%) & p-valor & OR $^{c}$ & IC $95 \%$ \\
\hline \multirow[t]{3}{*}{ Município de moradia } & Niterói & $25(11,0 \%)$ & $123(54,2 \%)$ & & & \\
\hline & Fora de Niterói & $14(6,2 \%)$ & $65(28,6 \%)$ & 0,86 & 1,06 & $0,52-2,18$ \\
\hline & Total & $39(17,2 \%)$ & । $88(82,8 \%)$ & & & \\
\hline \multirow[t]{3}{*}{ Idade materna } & $\geq 18$ & $29(12,8 \%)$ & $178(78,4 \%)$ & & & \\
\hline & $<18$ & $10(4,4 \%)$ & $10(4,4 \%)$ & $<0,01$ & 0,16 & $0,06-0,43$ \\
\hline & Total & $39(17,2 \%)$ & । $88(82,8 \%)$ & & & \\
\hline \multirow[t]{3}{*}{ Escolaridade } & Ensino Médio & $16(7,0 \%)$ & I I 8 (52,0\%) & & & \\
\hline & Ensino Fundamental & $23(10,1 \%)$ & $70(30,8 \%)$ & 0,02 & 0,41 & $0,20-0,83$ \\
\hline & Total & $39(17,2 \%)$ & । $88(82,8 \%)$ & & & \\
\hline \multirow[t]{3}{*}{ Número de gestações } & | gestação & $26(11,5 \%)$ & $76(33,5 \%)$ & & & \\
\hline & > I gestação & $13(5,7 \%)$ & $112(49,3 \%)$ & $<0,01$ & 0,34 & $0,16-0,70$ \\
\hline & Total & $39(17,2 \%)$ & $188(82,8 \%)$ & & & \\
\hline \multirow[t]{3}{*}{ Local de nascimento } & Baixa complexidade & $7(3,1 \%)$ & $72(31,7 \%)$ & & & \\
\hline & Alta complexidade & $32(14,1 \%)$ & | $16(5 \mid, 1 \%)$ & 0,02 & 2,84 & $1,19-6,77$ \\
\hline & Total & $39(17,2 \%)$ & । $88(82,8 \%)$ & & & \\
\hline \multirow[t]{3}{*}{ Número de consultas no pré-natal } & $<6$ & $15(6,6 \%)$ & $48(21,1 \%)$ & & & \\
\hline & $\geq 6$ & $24(10,6 \%)$ & $140(61,7 \%)$ & 0,12 & 0,55 & $0,27-1,13$ \\
\hline & Total & $39(17,2 \%)$ & I $88(82,8 \%)$ & & & \\
\hline \multirow[t]{3}{*}{ Parto } & Pélvico & $19(8,4 \%)$ & $85(37,4 \%)$ & & & \\
\hline & Cesariana & $20(8,8 \%)$ & $103(45,4 \%)$ & 0,73 & 0,87 & $0,44-1,73$ \\
\hline & Total & $39(17,2 \%)$ & I $88(82,8 \%)$ & & & \\
\hline
\end{tabular}

${ }^{a} \mathrm{SC}$ - sem conhecimento; ${ }^{\mathrm{b}} \mathrm{CC}$ - com conhecimento; $\mathrm{c} O \mathrm{R}$ - razão de chance; ${ }^{\mathrm{I} C} \mathrm{C}$ - intervalo de confiança.

Tabela 2 - Características das puérperas no estudo de variáveis contínuas que influenciam a falta de conhecimento materno sobre a triagem neonatal em Niterói (2013-2015)

\begin{tabular}{|c|c|c|c|c|c|c|c|c|c|}
\hline \multirow{2}{*}{ Variáveis contínuas } & \multicolumn{2}{|c|}{ Média } & \multicolumn{2}{|c|}{ IC $^{\mathrm{a}}(95 \%)$} & \multicolumn{2}{|c|}{$\mathbf{D P}^{\mathbf{b}}$} & \multicolumn{2}{|c|}{ MIN. - MÁX.c } & \multirow[b]{2}{*}{ p-valor } \\
\hline & $\mathbf{S C}^{\mathrm{d}}$ & $\mathbf{C C}^{\mathrm{e}}$ & $\mathbf{S C}^{\mathrm{d}}$ & $\mathbf{C C}^{\mathrm{e}}$ & $\operatorname{SC}^{d}$ & $\mathbf{C C}^{\mathrm{e}}$ & $\operatorname{sed}^{d}$ & $\mathrm{CC}^{\mathrm{e}}$ & \\
\hline Idade materna & 21,50 & 25,57 & $19,95-23,05$ & $24,66-26,48$ & 4,73 & 6,17 & $14-32$ & $14-46$ & $<0,01$ \\
\hline Número de filhos & 1,47 & 1,99 & $1,19-1,76$ & $1,83-2,15$ & 0,86 & I,II & $1-4$ & $1-7$ & $<0,01$ \\
\hline Número de gestações & ।,7। & 2,27 & $|, 3|-2,||$ & $2,05-2,48$ & $1,2 \mid$ & 1,46 & $1-6$ & $1-9$ & $<0,01$ \\
\hline Número de consultas no pré-natal & 6,50 & 7,43 & $5,63-7,37$ & $6,99-7,86$ & 2,64 & 2,95 & $0-12$ & $0-16$ & 0,14 \\
\hline Peso & $3.087,34$ & $3.158,17$ & $2.916,81-3.257,88$ & $3.081,98-3.234,36$ & 518,83 & 515,08 & $2.030-4.000$ & $1.415-4.700$ & 0,47 \\
\hline Idade gestacional & 38,64 & 41,09 & $38,13-39,15$ & $37,24-44,95$ & 1,54 & 26,06 & $33,6-41,1$ & $31,4-42,5$ & 0,02 \\
\hline Apgar $5^{\circ}$ minuto & 9,00 & 9,10 & $8,68-9,32$ & $8,97-9,22$ & 0,96 & 0,84 & $5-10$ & $3-10$ & 0,55 \\
\hline
\end{tabular}

${ }^{a} \mathrm{C}$ - intervalo de confiança; b DP - desvio-padrão; ‘MIN.-MÁX. - valor mínimo e máximo; ${ }^{\circledR} S C$ - sem conhecimento; ${ }^{e} C C$ - com conhecimento. 
Universidade de Chicago, revelou que apenas $35 \%$ das mulheres relataram ter recebido informações durante os cuidados pré-natais. ${ }^{18}$

No presente estudo, a maioria das mães classificadas como SC teve um perfil de idade jovem (menos de 18 anos), primigesta e poucos anos de estudo formal, além de ter seu parto realizado em unidade de alta complexidade. Um perfil semelhante foi encontrado em um estudo que avaliou a falta de conhecimento das doenças detectadas pelo rastreio neonatal entre jovens adultos. ${ }^{19}$

Estes resultados alertam para a necessidade de aumentar a conscientização em relação às intervenções preventivas. É essencial que a puérpera conheça e compreenda a importância do sistema de rastreio neonatal, uma vez que frequentemente é atribuída a ela a responsabilidade de levar o seu recém-nascido para os serviços de saúde locais para realizar a TN durante o período ideal, após receber alta da maternidade. Achados semelhantes foram encontrados nos Estados Unidos, onde a propagação de informações sobre o sistema de rastreamento neonatal e as amostras coletadas para tais testes foram consideradas inadequadas. ${ }^{20} \mathrm{De}$ acordo com o American College of Medical Genetics, é importante que os prestadores de cuidados à saúde e o público em geral sejam informados sobre a $\mathrm{TN}^{21}{ }^{21}$ especialmente no momento em que a possibilidade de sequenciamento genômico do material recolhido para rastreio está sendo discutida. ${ }^{22}$ As mulheres grávidas deveriam aprender que locais fornecem serviços de TN antes mesmo do parto. Essa informação deve ser prestada durante o pré-natal, visto que este tem sido descrito como um ambiente adequado para transmitir informações aos futuros pais. ${ }^{23}$ Curioso foi perceber que, entre as oito mulheres que declararam não ter passado pelo sistema de atendimento pré-natal, somente uma afirmou não ter conhecimento sobre a TN. Uma possível explicação vem do fato de que nesse grupo de sete puérperas seis tiveram dois ou mais filhos, passando a ideia de que a repetição de gestações pode interferir na geração do conhecimento sobre esse sistema de prevenção.

Além disso, as maternidades deveriam ser equipadas com a infraestrutura e a equipe necessárias para implementar o rastreio eficaz. Não deve haver margem para que crianças assintomáticas afetadas por doenças detectáveis pela TN não sejam diagnosticadas precocemente por meio desse teste.

Por outro lado, os esforços para aumentar a conscientização sobre o rastreamento neonatal não devem ser limitados aos cuidados maternos e pediátricos. Tal informação deve ser fornecida ao público em geral desde a escola primária, para garantir que esteja firmemente enraizada na mente dos futuros pais. Recentemente, vídeos educativos e fóruns on-line se tornaram ferramentas úteis para fornecer informações e orientação sobre o rastreamento de recém-nascidos. ${ }^{24,25}$

Os sistemas brasileiros de educação e de saúde são deficientes. Os achados deste estudo mostram que as instituições de saúde não conseguem estabelecer uma informação proativa e importante sobre o rastreamento neonatal, uma intervenção que pode detectar doenças de difícil controle antes mesmo do início dos sintomas. ${ }^{26}$ Também deve-se ter em mente que a demora no retorno do resultado é um problema real que atinge todo o sistema de rastreamento biológico. A valorização desse sistema deveria ser uma questão prioritária na saúde pública. Uma possível solução para esse cenário desarmônico seria a aplicação de educação em saúde, gerando informação pelas equipes de saúde aos pais (especialmente no pré-natal) e também aos gestores de todo o sistema.

\section{CONCLUSÕES}

Concluímos que as puérperas que demonstraram falta de conhecimento sobre a triagem neonatal eram, na maioria, jovens, em suas primeiras gestações, com baixo nível de escolaridade e em maternidades de alta complexidade. Assim, deve ser dada maior atenção à conscientização da TN neste grupo de mulheres. A falha do pré-natal na passagem de informações sobre o tema também foi evidente. Portanto, apesar do grande progresso alcançado no programa brasileiro de TN, ainda são necessários incentivos governamentais adicionais para otimizar sua eficiência.

\section{AGRADECIMENTOS}

À Universidade Federal Fluminense, agradecemos o incentivo e o apoio à pesquisa. 


\section{REFERÊNCIAS}

1 Brasil. Triagem neonatal biológica: manual técnico. Brasília: Ministério da Saúde; 2016. $80 \mathrm{p}$.

2 Leão L, Aguiar M. Newborn screening: what pediatricians should know. Jornal de Pediatria. 2008;84(4):s80-s90.

3 Leonard JV, Morris AA. Diagnosis and early management of inborn errors of metabolism presenting around the time of birth. Acta Paediatr. 2006;95(1):6-14.

4 Poretti A, Blaser SI, Lequin MH, Fatemi A, Meoded A, Northington FJ et al. Neonatal neuroimaging findings in inborn errors of metabolism. JJ Magn Reson Imaging. 2013;37(2):294-312.

5 Rubio-Agusti I, Carecchio M, Bhatia KP, Kojovic M, Parees I, Chandrashekar HS et al. Movement disorders in adult patients with classical galactosemia. Mov Disord. 2013;28(6):804-10.

6 Guthrie R, Susi AS. A simple phenylalanine method for detecting phenylketonuria in large populations of newborn infant. Pediatrics. 1963;32:338-43.

7 de Carvalho TM, dos Santos HP, dos Santos IC, Vargas PR, Pedrosa J. Newborn screening: a national public health programme in Brazil. J Inherit Metab Dis. 2007;30(4):615.

8 Souza CF, Schwartz IV, Giugliani R. Triagem neonatal de distúrbios metabólicos. Ciênc Saúde Coletiva. 2002;7(1):129-37.

9 Botler J, Camacho LAB, Cruz MM, George P. Triagem neonatal: o desafio de uma cobertura universal e efetiva. Ciênc Saúde Coletiva. 2010;15(2):493-508.

10 Mendes LC, dos Santos TT, Bringel FA. Evolution of the neonatal screening program in the state of Tocantins. Arq Bras Endocrinol Metab. 2013;57(2):112-9.
11 Brasil. Teste do pezinho deve ser feito até o $5^{\circ}$ dia de vida do recém-nascido. Brasília: Ministério da Saúde; 2017 [acesso em: 22/11/2017]. Disponível em: http://www. brasil.gov.br/saude/2017/06/teste-dopezinho-deve-ser-feito-ate-o-5-dia-devida-do-recem-nascido

12 American College of Obstetricians and Gynecologists' Committee on Practice Bulletins - Obstetrics; Committee on Genetics; Society for MaternalFetal Medicine. Practice bulletin n. 162: prenatal diagnostic testing for genetic disorders. Obstet Gynecol. 2016;127(5):e108-22.

13 Pena SD, Bastos-Rodrigues L, Pimenta JR, Bydlowski SP. DNA tests probe the genomic ancestry of Brazilians. Braz J Med Biol Res. 2009;42(10):870-6.

14 Haidar FH, Oliveira UF, Nascimento LF. Maternal educational level: correlation with obstetric indicators. Cadernos Saúde Pública. 2001;17(4):1025-9.

15 Al-Sulaiman A, Kondkar AA, Saeedi MY, Saadallah A, Al-Odaib A, Abu-Amero KK. Assessment of the knowledge and attitudes of Saudi mothers towards newborn screening. BioMed Research International. 2015;2015:718674.

16 Burlina AB, Corsello G. Survey of Italian pediatricians' perspectives and knowledge about neonatal screening. Ital J Pediatr. 2015;41:41.

17 Committee on Genetics. Committee opinion n. 616: newborn screening and the role of the obstetrician-gynecologist. Obstet Gynecology. 2015;125(1):256-60.

18 Lang CW, Stark AP, Acharya K, Ross LF. Maternal knowledge and attitudes about newborn screening for sickle cell disease and cystic fibrosis. Am J Med Genet A. 2009;149A(11):2424-9.

19 Noke M, Ulph F. Young adults' pre-existing knowledge of cystic fibrosis and sickle cell diseases: implications for newborn screening. J Genetic Couns. 2014;23(1):121-30.

20 Newcomb P, True B, Walsh J, Dyson M, Lockwood S, Douglas B. Maternal attitudes and knowledge about newborn screening. MCN Am J Matern Child Nurs. 2013;38(5):289-94.

21 Watson MS, Mann MY, Lloyd-Puryear MA, Rinaldo P, Howell RR. American College of Medical Genetics Newborn Screening Expert Group. Newborn screening: toward a uniform screening panel and system - Executive summary. Pediatrics. 2006;117:S296-S307.

22 Joseph G, Chen F, Harris-Wai J, Puck JM, Young C, Koenig BA. Parental views on expanded newborn screening using whole-genome sequencing. Pediatrics. 2016;137(Suppl 1):S36-S46.

23 Botkin JR, Rothwell E, Anderson RA, Rose NC, Dolan SM, Kuppermann M et al. Prenatal education of parents about newborn screening and residual dried blood spots: a randomized clinical trial. JAMA Pediatr. 2016;170(6):543-9.

24 Strekalova YA. Finding motivation: online information seeking following newborn screening for cystic fibrosis. Qual Health Res. 2016;26(9):1180-90.

25 Temme R, Gruber A, Johnson M, Read L, Lu Y, McNamara J. Assessment of parental understanding of positive newborn screening results and carrier status for cystic fibrosis with the use of a short educational video. J Genet Couns. 2015;24(3):473-81.

26 Brousseau T, Sharieff GQ. Newborn emergencies: the first 30 days of life. Pediatr Clin North Am. 2006;53(1)69-84. 